\title{
Effects of Tube Voltage on Phase-Contrast Imaging for Different Microfocus X-Ray Tubes
}

\author{
Jianbao Gui, Zhanli Hu, Peter Z. Wu, Hairong Zheng \\ Paul C. Lauterbur Research Center for Biomedical Imaging, Institute of Biomedical and Health Engineering, \\ Shenzhen Institutes of Advanced Technology, Chinese Academy of Sciences, Shenzhen, China \\ Email: jb.gui@siat.ac.cn, hr.zheng@siat.ac.cn
}

Received 2013

\begin{abstract}
In the past decade, phase-contrast imaging (PCI) has become a hot research with an increased improvement of the image contrast with respect to conventional absorption radiography. In this paper, effects of tube voltage (kVp) on propagation-based phase-contrast imaging have been investigated with two types of microfocus $\mathrm{x}$-ray tubes, a conventional sealed x-ray tube with the focal spot size of $13-20 \mu \mathrm{m}$ and an open x-ray tube with minimum focal spot size less than 2 $\mu \mathrm{m}$. A cooled x-ray CCD detector with the pixel size of $24 \mu \mathrm{m}$ was used to acquire digital images. Two thin plastic sheets with different thickness were used as radiography phantoms. Two different phenomena were observed for the two x-ray tubes. For the open tube, phase-contrast effect has a slight drop with the increasing of tube voltage, however, it is opposite for the sealed tube. A further investigation indicates that the variation of focal spot size causes the abnormal result for the sealed tube. It also shows that phase-contrast effect is more sensitive to focal spot size than tube voltage.
\end{abstract}

Keywords: Phase-Contrast Imaging; Edge Enhancement; X-Ray; Tube Voltage

\section{Introduction}

Conventional $\mathrm{x}$-ray imaging is based on the attenuation properties of an object, however, for some weak absorption objects, such as biological tissue, polymers, and fiber composites, the use of conventional $\mathrm{x}$-ray imaging is limited due to poor image contrast. While the application of $\mathrm{x}$-ray phase-contrast imaging can provide a way to generate the images with better image-contrast because the interaction cross-section of $\mathrm{x}$-ray phase-shift is about a thousand times larger than that of absorption for soft tissues [1].

Wilkins and his colleague firstly developed a classical theory formula (PGW theory) [2] on propagation-based phase-contrast imaging (PB-PCI) in 1997. This PGW theory is based on the paraxial Fresnel-Kirchhoff diffraction for spatially coherent x-ray sources. In 2004, Wu and Liu publicized a more comprehensive theory based on Wigner distribution function (WDF) [3], which can deal with partiallycoherent x-ray and the detector's impact. According to the PGW theory, for a weak absorption and weak phase-shift object,

\footnotetext{
"This work was supported in part by National Science \& Technology Pillar Program (2012BAI13B04), National Natural Science Foundation of China (81401410), Special Fund Project and Basic Research Program of Shenzhen (CXZZ20140505091419405, JCYJ201304011703 06796, JCYJ20140417113430558, JCYJ20130401170306880).
}

$$
I(x) \approx 1-\frac{\lambda^{2}}{2 \pi} r_{e} z \rho_{e}^{\prime \prime}(x),
$$

where $r_{e}$ is the classical electron radius and $\rho_{e}$ the projected electron density, $\lambda$ denotes the wavelength and $\mathrm{z}$ the propagation distance. From the formula, we can know that a longer wavelength and a larger propagation distance are helpful to the forming of phase contrast, which means a lower $x$-ray energy expected.

We can also consider phase-contrast effect from lateral coherence length for the PB-PCI depending on a partially coherence microfocus $\mathrm{x}$-ray source,

$$
d_{\perp}=\lambda R_{1} / \sigma=\lambda / \gamma,
$$

where $\sigma$ is the source size, $\gamma$ is the angular width of the source as viewed from the observation point. The lateral coherence length is larger, phase-contrast effect is better. From this way, we can see that longer wavelengths (lower x-ray energies), longer SOD distances and smaller focus-spot sizes, are beneficial for the phase-contrast imaging.

Donnelly investigated some influencing factors by experiments based on different PCI systems [4-6]. It is interesting that Donnelly showed that phase enhancement effect had a slightly drop with the increasing of tube voltage $(\mathrm{kVp})$ in this paper [4], but the author also showed a 
different result that phase enhancement effect essentially wasn't affected as the tube voltage increased in other two papers $[5,6]$.

In this paper, we had a detail experimental investigation on the effect of tube voltage on phase-contrast imaging. This study was based on two types of x-ray tubes, a sealed microfocus x-ray tube and an openmicrofocus $\mathrm{x}$-ray tube, and the later has some better properties suitable for PCI study, for example, smaller and adjustable focal spot sizes, higher $\mathrm{kVps}$ and higher powers. The study is significant for optimizing system design and exploring common rules of the PB-PCI technology.

\section{Materials and Methods}

Two investigations were performed based on a conventional sealed x-ray source (UltraBright, Oxford Instruments) and an open x-ray source (FXE 160.51, YXLON), respectively. The sealed $\mathrm{X}$-ray source has a focal spot size of $13-20 \mu \mathrm{m}$, tube voltage and tube power can been adjusted in the range of $20-90 \mathrm{kVp}$ and $10-19 \mathrm{~W}$, respectively. The open x-ray source has a minimum focal spot size less than $2 \mu \mathrm{m}$ and the focal spot size can been adjusted by changing the focusing current, which is the current flowing through the focusing lenses. The tube voltage $(\mathrm{kVp})$ can be varied from 20 to $160 \mathrm{kVp}$, maximum tube power and target power is $64 \mathrm{~W}$ and $10 \mathrm{~W}$, respectively. A cooled x-ray CCD imaging detector (Quad-RO 4320, Princeton Instruments) was used to acquire the images. The CCD imaging detector has a high spatial resolution, large active imaging area and low noise $(24 \mu \mathrm{m}$ pixel size, $2084 \times 2084$ array and $50 \times 50$ $\mathrm{mm}^{2}$ active area, ultra low noise electronics and state-ofthe-art cooling technology), these characteristics are also helpful for phase-contrast imaging investigation. The sourceto-detector distance (SDD) was held at $710 \mathrm{~mm}$ and the SOD could be adjusted from 10 to $510 \mathrm{~mm}$.

In order to quantify the edge enhancement effect of phase-contrast imaging, we adopted the same edge enhancement index (EEI) as Donnelly [4]. The EEI is defined as follows:

$$
E E I=\frac{(P-T) /(P+T)}{(H-L) /(H+L)},
$$

where $P$ and $T$ are the peak and trough intensity values at the edge, and $H$ and $L$ are average values of multi-pixels on the high- and low-intensity regions next to the edge.

Two other indexes [4] were also evaluated: the upstroke index (UI), defined as follows:

$$
U I=(P-H) /(P+H),
$$

and the downstroke index $(D I)$, defined as follows:

$$
D I=(L-H) /(L+H) .
$$

For the sealed x-ray tube, we have performed the im- aging at different $\mathrm{x}$-ray tube voltages with constant tube power of $10 \mathrm{~W}$. In order to focus on the edge-enhanced phenomenon, a thin rectangular plastic sheet of $0.5 \mathrm{~mm}$ thickness was used as a radiography phantom. The SOD was kept at $185 \mathrm{~mm}$ and exposure time was $8 \mathrm{~s}$.

For the open x-ray tube, to evaluate the effect of the tube voltage, two sets of experiments were performed. In the first one, the target power was held constant at $3 \mathrm{~W}$ and the tube voltage was adjusted. In the second one, target current was held constant at $30 \mu \mathrm{A}$ and the tube voltage was adjusted. The SOD was held at $40 \mathrm{~mm}$ and exposure time was adjusted to maintain an approximate constant exposure intensity. A plastic sheet of $2 \mathrm{~mm}$ thickness was used as a radiography phantom.

The image at each $\mathrm{kVp}$ was obtained by averaging 5 frames acquired continuously and profiles of pixel intensity values were obtained by averaging 50 continuous rows to reduce noise. Quantitative indexes $U I, D I$ and EEI were computed for all images.

\section{Results and Discussions}

\subsection{Sealed X-Ray Tube}

Depending on the sealed type x-ray tube (UltraBright, Oxford Instruments), the phase-contrast image of the 0.5 mm thickness plastic phantom was acquired at a representative tube voltage of $50 \mathrm{kVp}$, as shown in Figure 1(a). From the image we can see obvious edge-enhancement (high- and low-intensity vertical lines) at two edges of the phantom. The profiles along the middle horizontal line of the images obtained at different tube voltages are shown in Figure 1(b). It is interesting to note that this effect is more pronounced at a higher $\mathrm{kVp}$ value. The quantitative upstroke index shows the same result that edge enhancement becomes more pronounced as the $\mathrm{kVp}$ increases.

It is contrary to the common belief that better phasecontrast occurs at the lower energy since the longer wavelength of x-ray photos at a lower tube voltage can improve the phase-contrast. Fortunately, the variation of focal-spot size at different operation conditions, which is observed in the other experiments, inspires us to think about the effect focal-spot size on the phase-contrast imaging.

In order to quantify the size of the focal spot in our system, we imaged a JIMA (Japan Inspection Instruments Manufacturers' Association) resolution test-pattern with the micro-focus x-ray system. By using the resolution test-pattern, the acquired images at different tube voltages are shown in Figure 2, from which it is seen that the spatial resolution of the images becomes better with the increase of $\mathrm{kVp}$ value. It means that the variation of focal-spot size causes the abnormal phenomenon, though a higher tube voltage can decrease the edge en- 
hancement, the effect of focal-spot size is greater. From our results, it can be derived that phase-contrast effect is insensitive to tube voltage (or equivalently, wavelength of x-ray photos), but sensitive to focal-spot size.

\subsection{Open X-Ray Tube}

\subsubsection{Constant Target Power}

Figure 3(a) shows the phantom image obtained at 80 $\mathrm{kVp}$ when a constant target power of $3 \mathrm{~W}$ was held. Representative profiles of the pixel intensity values across the edge images obtained at different tube voltages are shown in Figure 3(b). EEI as a function of $\mathrm{kVp}$ are shown in Figure 3(c). These indicate a different re- sult that edge enhancement drops as $\mathrm{kVp}$ increases. In Figure 4, images of JIMA resolution test-pattern show that the spatial resolutions are almost same at different $\mathrm{kVp}$.

\subsubsection{Constant Target Current}

Whena constant target current was held, representative profiles of the pixel intensity values across the edge images obtained at different tube voltages are shown in Figure 5(a). EEI as a function of tube voltage are shown in Figure 5(b). These results also show that edge enhancement has a drop as the kVp increases. In Figure 6, images of JIMA resolution test-pattern shows the spatial resolutions are almost same at different $\mathrm{kVp}$.

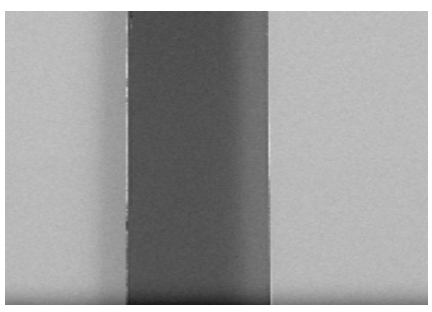

(a)

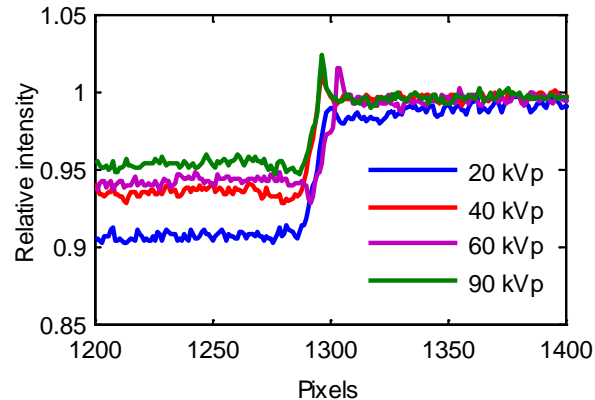

(b)

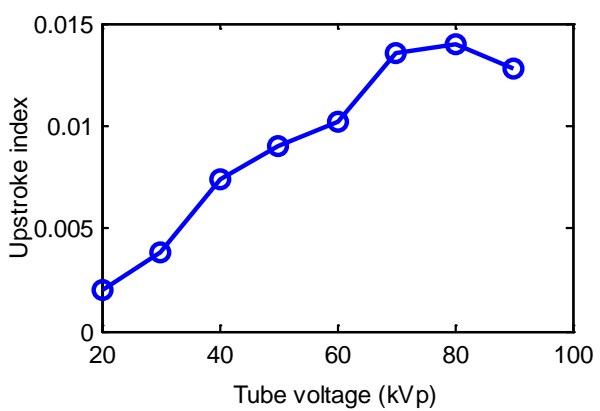

(c)

Figure 1. (a) The $0.5 \mathrm{~mm}$ plastic edge phantom image obtained at $50 \mathrm{kVp}$ and the SOD of $185 \mathrm{~mm}$, (b) representative profiles near to the right edge at different tube voltages and (c) EEI as a funtion of $\mathrm{kVp}$. The tube power was held constant at $10 \mathrm{~W}$. This result was obtained based on the sealed type $x$-ray tube (UltraBright, Oxford Instruments).

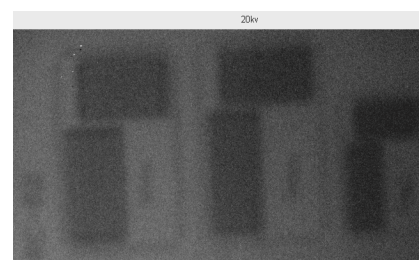

(a)

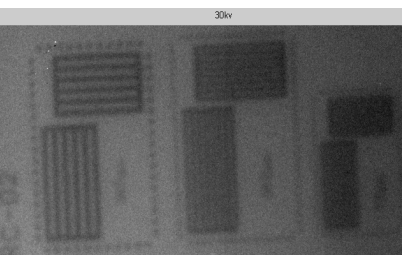

(b)

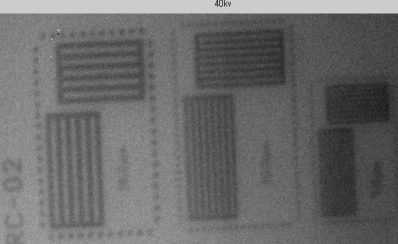

(c)

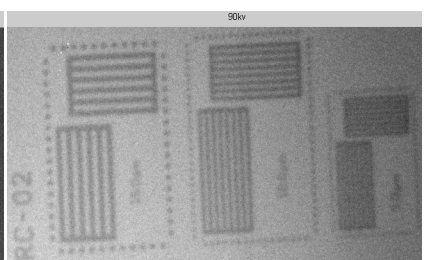

(d)

Figure 2. Representative images of the JIMA resolution test- pattern at different tube voltage, $20 \mathrm{kVp}, 30 \mathrm{kVp}, 40 \mathrm{kVp}$ and 90 $\mathrm{kVp}$, display window is $[0.9,1.5]$. All images were acquired at the tube power of $10 \mathrm{~W}$. The resolution pattern from left to right in every figure is 15, 10 and $7 \mu \mathrm{m}$, respectively. (a) $20 \mathrm{kVp}, 10 \mathrm{~W}$; (b) $30 \mathrm{kVp}, 10 \mathrm{~W}$; (c) $40 \mathrm{kVp}, 10 \mathrm{~W}$; (d) $90 \mathrm{kVp}, 10 \mathrm{~W}$.

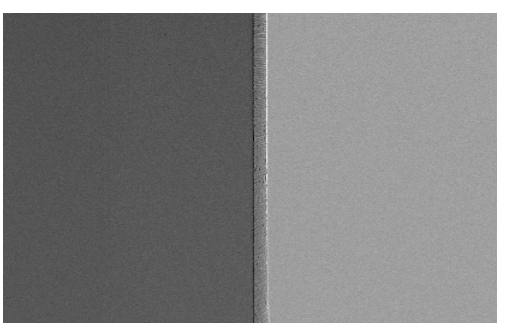

(a)

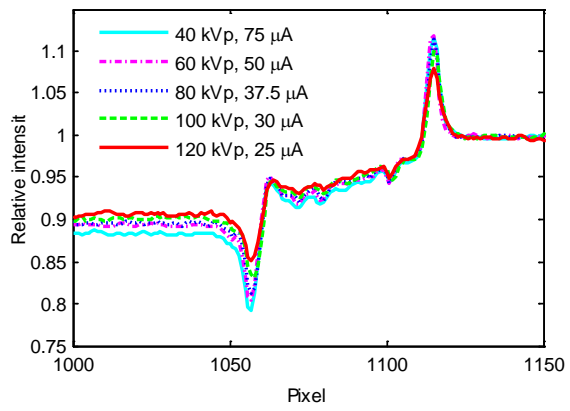

(b)

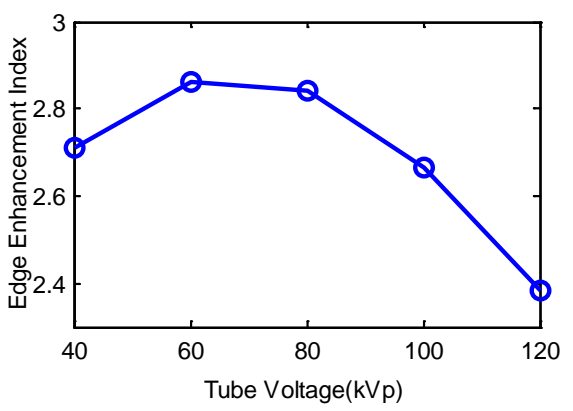

(c)

Figure 3. (a) The $2 \mathrm{~mm}$ plastic edge phantom image obtained at $80 \mathrm{kVp}$ and the SOD of $40 \mathrm{~mm}$, (b) representative profiles near to the edge at different tube voltages and (c) $E E I$ as a funtion of $\mathrm{kVp}$. The tube power was held constant at $3 \mathrm{~W}$. This result was obtained based on the open type $x$-ray tube (FXE 160.51, YXLON). 


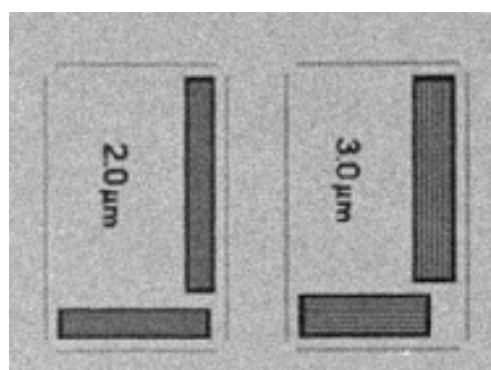

(a)

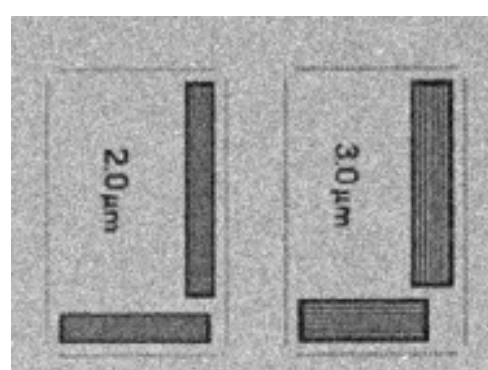

(b)

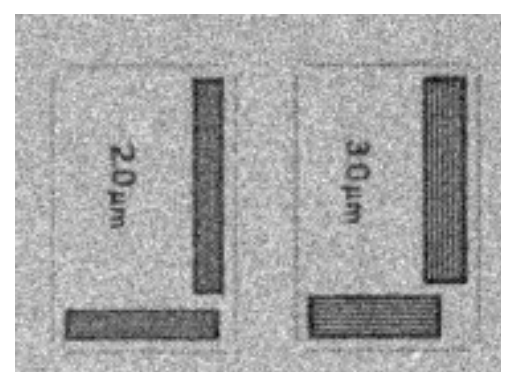

(c)

Figure 4. Images of JIMA resolution test-pattern at different tube voltages with constant target power, (a) $40 \mathrm{kVp},(\mathrm{b}) 80 \mathrm{kVp}$, (c) $120 \mathrm{kVp}$. All images show the same spatial resoltion of $3 \mu \mathrm{m}$.

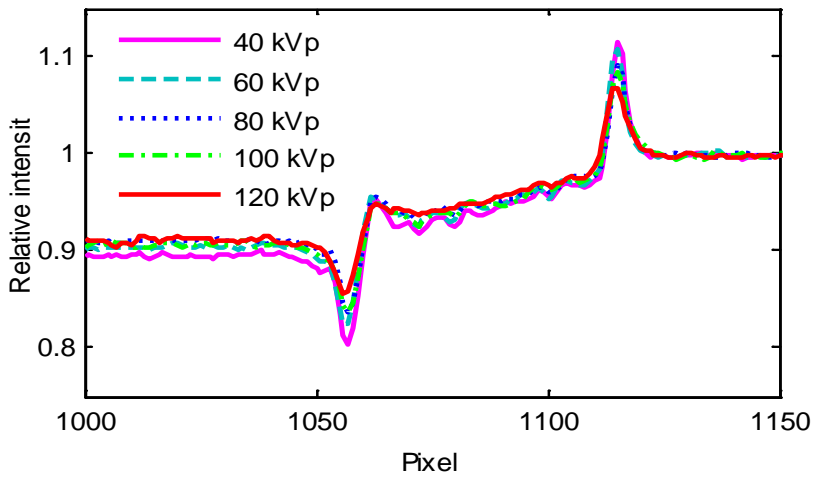

(a)

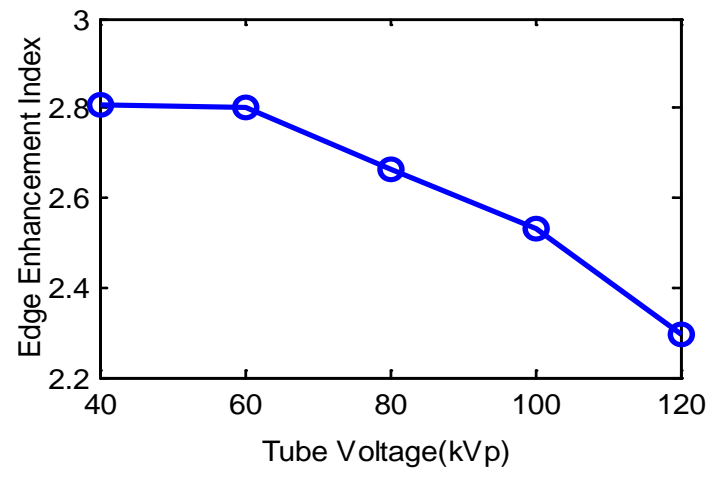

(b)

Figure 5. (a) Representative profiles near to the edge at different tube voltages and (b) $E E I$ as a funtion of $\mathrm{kVp}$. The target current was held constant at $30 \mu \mathrm{A}$. This result was obtained based on the open type x-ray tube (FXE 160.51, YXLON).

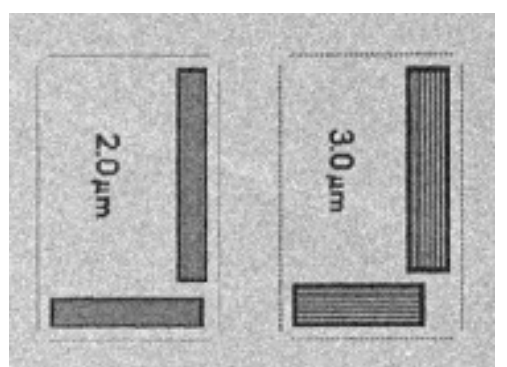

(a)

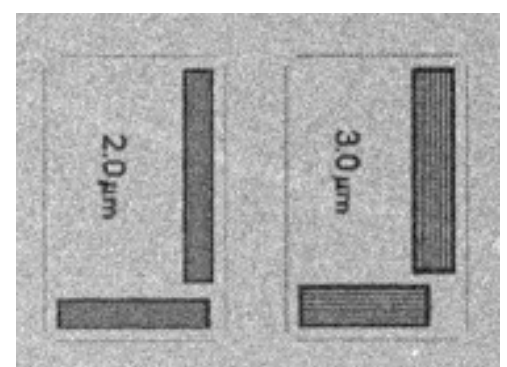

(b)

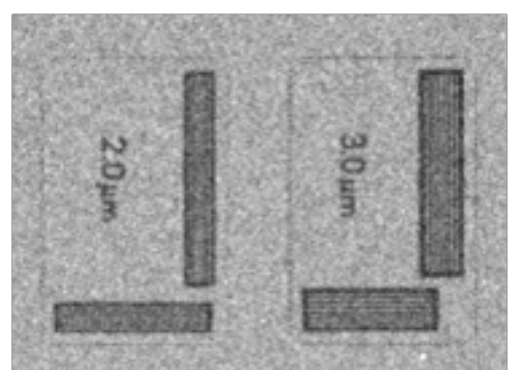

(c)

Figure 6. Images of JIMA resolution test-pattern at different tube voltage with constant target current, (a) $40 \mathrm{kVp}$, (b) 80 kVp and (c) $120 \mathrm{kVp}$. All images show the same spatial resoltion of $3 \mu \mathrm{m}$.

Both the study at constant target power and the study at constant target current demonstrate that edge enhancement has a drop with the increasing of tube voltage, but the drop is slight since the edge enhancement at the high voltage of $120 \mathrm{kVp}$ is still obvious. This result is consistent with the PGW theory and lateral coherence length formula, longer wavelength (lower x-ray energy) is beneficial for the phase-contrast imaging. The use of high tube voltages over $100 \mathrm{kVp}$ is very significant in reducing patient dose if the phase-contrast imaging technology will be applied in human breast diagnosis in the future.

\section{Conclusion}

We investigated the effect of tube voltage $(\mathrm{kVp})$ on the propagation-based phase-contrast imaging with two types of microfocus $\mathrm{x}$-ray tubes, a conventional sealed $\mathrm{x}$-ray tube and an open $x$-ray tube. Two different phenomena were observed for the two x-ray tubes. For the open tube, phase-contrast effect has a slight drop with the increasing of tube voltage, however, it is opposite for the sealed tube. A further investigation indicates that the variation of focal spot size causes the abnormal result for the sealed tube. It also shows that phase-contrast effect is more sensitive to focal spot size than tube voltage. 


\section{REFERENCES}

[1] X. Z. Wu and H. Liu, "Clinical Implementation of X-Ray Phase-Contrast Imaging: Theoretical Foundations and Design Considerations,” Medical Physics, Vol. 30, No. 8, 2003, pp. 2169-2179.

http://dx.doi.org/10.1118/1.1593836

[2] A. Pogany, D. Gao and S. W. Wilkins, "Contrast and Resolution in Imaging with a Microfocus X-Ray Source,” Review of Scientific Instruments, Vol. 68, No. 7, 19997, pp. 2774-2782. http://dx.doi.org/10.1063/1.1148194

[3] X. Z. Wu and H. Liu, "Phase-Space Formulation for PhaseContrast X-Ray Imaging,” Applied Optics, Vol. 44, No. 28, 2005, pp. 5847-5854.

http://dx.doi.org/10.1364/AO.44.005847
[4] F. D. Edwin and R. P. Ronald, "Quantification of the Effect of kVp on Edge-Enhancement Index in Phase-Contrast Radiography,” Medical Physics, Vol. 29, No. 6, 2002, pp. 999-1002. http://dx.doi.org/10.1118/1.1477416

[5] F. D. Edwin, et al., "Characterization of the Phase-Contrast Radiography Edge-Enhancement Effect in a Cabinet X-Ray System,” Physics in Medicine and Biology, Vol. 51, No. 1, 2006, p. 21. http://dx.doi.org/10.1088/0031-9155/51/1/002

[6] F. D. Edwin, R. P. Ronald and R. P. David, "Quantification of the Effect of System and Object Parameters on Edge Enhancement in Phase-Contrast Radiography,” Medical Physics, Vol. 30, No. 11, 2003, pp. 2888-2896. http://dx.doi.org/10.1118/1.1617430 